\title{
Interface Sharpness in Amorphous Multilayer Heterostructures and their Effect on Quantum Confinement
}

Andrew Thron, Adam Schwartzberg and Shaul Aloni

${ }^{1}$ Molecular Foundry, Lawrence Berkeley National Laboratory, Berkeley, CA 94720

Atomic Layer Deposition (ALD) is advantageous over physical vapor depositions techniques due to its ability to control the deposition of materials one atomic layer at a time. Moreover, ALD's self-limiting growth mechanism and its chemical flexibility allow precise deposition of insulators, metals and semiconductors with unprecedented precision in thickness and composition. $\mathrm{TiO}_{2}$ has gained recent attention due to the wide variety of applications in solar energy conversion, such as water splitting, catalytic break down of organic pollutants, and solar cells. Quantum confinement was previously shown to occur in amorphous $\mathrm{TiO}_{2}$ films [1], and is suggested as means to extend $\mathrm{TiO}_{2}$ absorption from $\mathrm{UV}$ to the visible range of a spectrum. In order to increase the spectral range in which $\mathrm{TiO}_{2}$ can absorb light a multilayer structure can be created, where the thickness of alternating $\mathrm{TiO}_{2}$ layers is used to control its absorption. In this study the effect of interface sharpness on the optical absorption properties, in amorphous $\mathrm{TiO}_{2} / \mathrm{SiO}_{2}$ multilayer films, is investigated using scanning transmission electron microscopy (STEM), energy dispersive X-ray Spectroscopy (EDX), and Electron Energy Loss Spectroscopy (EELS).

$\mathrm{TiO}_{2} / \mathrm{SiO}_{2}$ multilayer films, nominally $2 \mathrm{~nm} / 10 \mathrm{~nm}$ thick, were created on $\mathrm{Si}$ (100) substrates using ALD. Optical absorptions measurements of the multilayered film showed a blue shift in the absorption edge of the $\mathrm{TiO}_{2}$, compared with that of a 40nm thick $\mathrm{TiO}_{2}$ film. Cross-Section samples were created using the standard lift-out technique in a focused ion beam microscope (FIB).

Amorphous nature of the layers has been confirmed through TEM and selected area diffraction (Figure 1). EDS and EELS spectral images were collected at 80 and 200kV, at ambient and 77K temperatures to study the effects of beam damage, beam spreading and radiation induced diffusion. An annular dark field (ADF) image in Figure 2a shows nine $\mathrm{TiO}_{2}$ layers, which are brightest in contrast. From the EDX line scans, a significant Si signal is detected within the nominal $\mathrm{TiO}_{2}$ layers, which may suggest intermixing of the layers (Figure $2 \mathrm{~b}$ ). ADF images and EELS line scans where acquired at $200 \mathrm{kV}$ to negate any artifacts caused by beam spreading (Figure 2). EELS line scans confirm that a $\mathrm{Si}$ signal is detected in the $\mathrm{TiO}_{2}$ layers, and that the signal is most significant in the bottom half of the layer. A close examination of $O$ concentrations profile in Figure $2 \mathrm{~d}$ reveals a similar trend to that of the Si profiles, which suggests that the top of the $\mathrm{SiO}_{2}$ layers are rough. This gives insight into the morphology of the film, but it is difficult to characterize the chemical sharpness of the $\mathrm{TiO}_{2} / \mathrm{SiO}_{2}$ interface.

Our TEM results suggest that $\mathrm{SiO} 2$ layers grow with a 2.3-3.0nm roughness under these conditions. Furthermore residual presence of $\mathrm{Si}$ in the $\mathrm{TiO} 2$ layers is associated either with long residency time of the $\mathrm{SiO} 2$ precursor or interdiffusion of $\mathrm{Si}$ into the $\mathrm{TiO}_{2}$ film. 


\section{References:}

[1] King D.M. et. al., Nanotechnology, 19 (2008), 445401
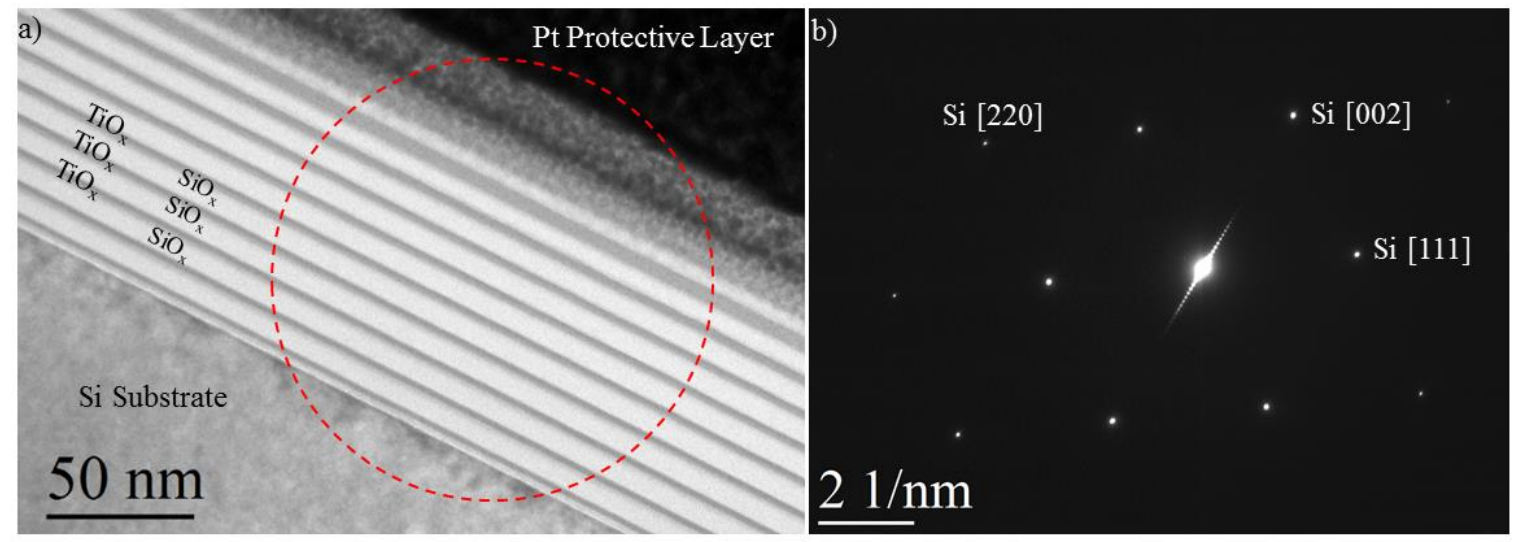

Figure 1. (a) TEM cross sectional, overview image of the $\mathrm{TiO}_{2} / \mathrm{SiO}_{2}$ Multilayer film on the Si substrate. (b) Selected Area Diffraction Pattern acquired from the area highlighted by the red circle in (a). Diffraction spots originate from the Si substrate. The diffraction pattern at the center of pattern originate from the multiple layers in the film.
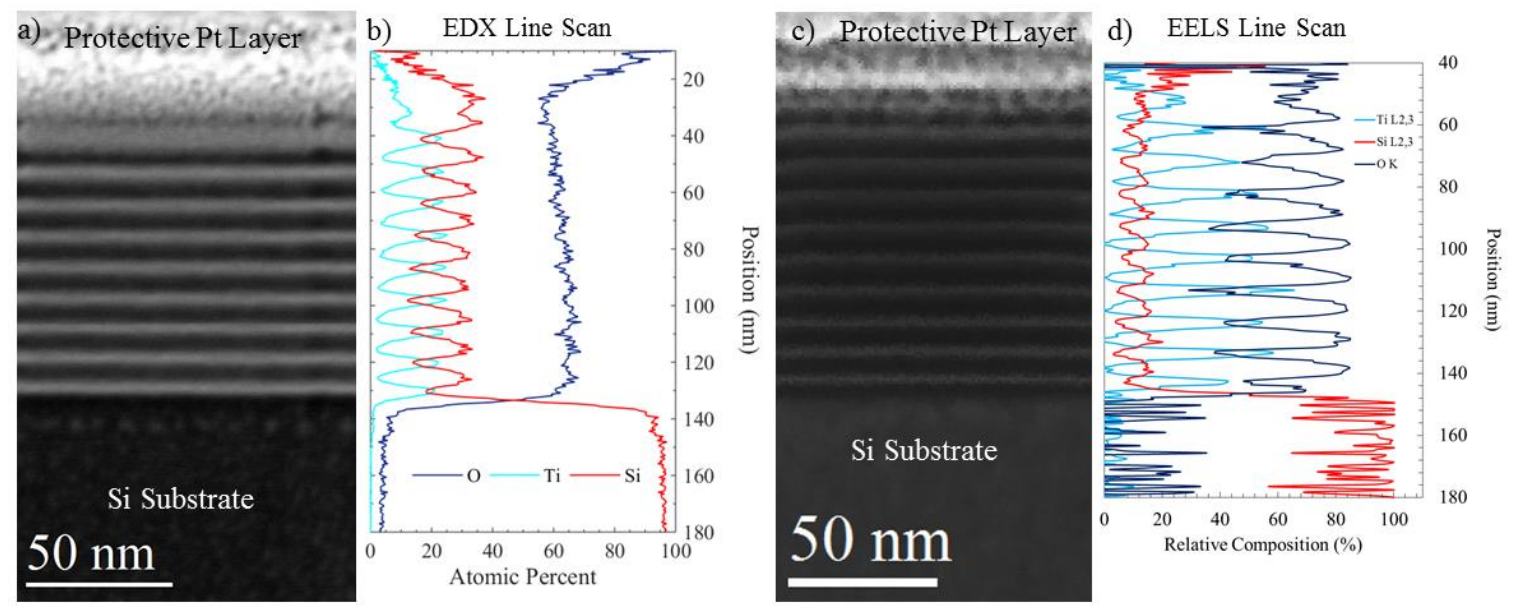

Figure 2. (a) ADF image of the multilayer film where the EDX spectra were acquired. (b) EDX line scan of the multi layered film showing the change in atomic percent of $\mathrm{Ti}, \mathrm{Si}$, and $\mathrm{O}$ across the film. (c) ADF image acquired from the area of the multilayer film where the EELS line scan was acquired. (d) Changes in the average, relative composition of the multilayer film, calculated from the EEL edges. 